\title{
Synthesis and Anticancer Activity of Some New Thiopyrano[2,3-d]thiazoles Incorporating Pyrazole Moiety
}

\author{
Nadia Hanafy Metwally,* Mohamed Ahmed Badawy, and Doha Samir Okpy
}

Chemistry Department, Faculty of Science, Cairo University; Giza 12613, Egypt.

Received December 25, 2014; accepted April 7, 2015

The knöevenagel condensation of 3-phenyl-4-thioxo-2-thiazolidinone (1) with 1-phenyl-3-aryl-1Hpyrazole-4-carbaldehydes $2 a-d$ in refluxing glacial acetic acid or in polyethylene glycol-400 (PEG-400) at room temperature without catalyst, afforded the corresponding 5-hetarylmethylene derivatives 3a-d. $[4+2]$ Cycloaddition reaction of compounds 3 with $N$-arylmaleimides, acrylonitrile and ethyl acrylate afforded thiopyrano $[2,3-d]$ thiazole derivatives $5 \mathrm{a}-\mathrm{p}$. The anticancer activity of some of the newly synthesized compounds was investigated against different human cancer cell lines (MCF7 and HEPG2) and confirmed by molecular docking. Moreover, the structure for one representative example of the new products was confirmed by X-ray crystallography. The structure of all the newly synthesized compounds was established by elemental and spectral data.

Key words 4-thioxo-2-thiazolidinone; thiopyrano[2,3-d]thiazole; anticancer activity

Thiazolidinones and their derivatives are an important group of heterocyclic compounds, having valuable biological activities in the areas of medicine and agriculture. ${ }^{1)}$ Moreover, thiazolidinone derivatives have several promising pharmacological actions such as antimicrobial, ${ }^{2-4)}$ anti-diabetic ${ }^{5-7)}$ anticonvulsant, ${ }^{8,9)}$ anticancer ${ }^{10,11)}$ activities, anti-inflammatory, ${ }^{12-14)}$ analgesic ${ }^{15,16)}$ anti yellow fever virus (YFV), ${ }^{17)}$ anti-human immunodeficiency virus (HIV) ${ }^{18)}$ and anti-tubercular. ${ }^{19)}$ Therefore, the investigation of chemistry and biology of these compounds continue to appeal the synthetic and medicinal organic researches. A majority of the biologically active thiazolidinone-derived compounds are 5-ylidene derivatives, probably because of the substituents at the C- 5 position of basic heterocycle which is crucial for the pharmacological effects. ${ }^{20-22)}$ Moreover, structure of the substituent in position C-5 of thiazolidinone cycle is determinative for the anticancer activity realization for all the hetaryl substituted thiazolidinones. That's why modification of mentioned position is the key concept of directed synthesis of novel anticancer agents in described class of compounds. These results prompted us to search for new 5-substituted-2-thiazolidinones of interest biological activity. In addition, thiopyrano[2,3- $d]$ thiazole derivatives considered as novel anticancer lead-compounds. ${ }^{23-26)}$ Also, pyrazoles have received much attention due to their diverse biological activities. ${ }^{27-29)}$ Our premise is that 2-thiazolidinones are synthetic precursors of thiopyrano[2,3-d]thiazole moiety, which could imitate some pharmacologically important molecular fragments of biologically active compounds. Based on the wide spectrum of biological profile of thiazolidinone, pyrazole and thiopyrano[2,3- $d]$ thiazole and their increasing importance in pharmaceutical, biological field and in continuation of our ongoing research on biologically active heterocycles, ${ }^{30-33)}$ it was thought of interest to accommodate thiopyrano[2,3-d]thiazole and pyrazole moieties in a single molecular frame work to synthesize some new tetracyclic fused heterocyclic compounds with potential anticancer activity. Molecular docking was performed for some selected compounds.

\section{Results and Discussion}

Chemistry The synthetic pathway that leads to the formation of the target 5-hetarylmethylene-4-thioxo-2-thiazolidinone 3a-d, were synthesized via knöevenagel condensation of 3-phenyl-4-thioxo-2-thiazolidinone (1) with each of 1-phenyl3-aryl-1H-pyrazole-4-carbaldehydes $\mathbf{2 a - d}$ in refluxing glacial acetic acid or in polyethylene glycol-400 (PEG-400) at room temperature without catalyst, as an efficient and simple green method in good yield (Chart 1). Elemental and spectral analyses were consistent with 5-hetarylmethylene-4-thioxo-2thiazolidinone 3a-d. The ${ }^{1} \mathrm{H}-\mathrm{NMR}$ spectrum of $\mathbf{3 d}$ displayed a singlet signal resonating at $\delta 8.17$ due to vinylic proton, in addition to the aromatic multiplets in the region $\delta 7.40-8.06$. The IR spectrum showed a carbonyl absorption band at $1712 \mathrm{~cm}^{-1}$ and its mass spectrum exhibited, among other fragments, ion peaks at $m / z 674\left(\mathrm{M}^{+}\right)$. From these data the $Z$-configuration was assigned to structures $\mathbf{3}$ on the basis that the olefinic proton of the $Z$-configured isomer is more deshielded by the thioxo group of the thiazole moiety compared with the $E$-isomer and appears at lower field ( $\delta c a .8 .0-8.50 \mathrm{ppm})$ relative to the $E$-isomer ${ }^{34,35)}$ ( $\left.\delta c a .7 .30-7.60 \mathrm{ppm}\right)$ ( $c f$. Chart 2, Experimental).

Next, when compounds $\mathbf{3 a}-\mathbf{d}$ were allowed reacting with $N$-arylmaleimides $\mathbf{4 a -}-\mathbf{d}$ in refluxing glacial acetic acid afforded in each case one adduct as evidenced by TLC. The products obtained were identified as 1:1 cycloadducts $\mathbf{5 a}-\mathbf{p}$ as depicted in Chart 1. The assigned structure for the isolated products $\mathbf{5 a}-\mathbf{p}$ was established on the basis of the elemental analysis and spectral data. The IR spectrum of 5i showed absorption bands at $v_{\max } 1718$ and $1689 \mathrm{~cm}^{-1}$ corresponding to carbonyl groups. Its ${ }^{1} \mathrm{H}-\mathrm{NMR}$ spectrum revealed two doublets at $\delta 4.35 \mathrm{ppm}$ with $J$ values $8.6,3.6 \mathrm{~Hz}$ corresponding to $\mathrm{H}-6$, a doublet signal at $\delta 4.52 \mathrm{ppm}$ with $J$ value $3.6 \mathrm{~Hz}$ attributed to $\mathrm{H}-5$ and a doublet signal at $\delta 4.95 \mathrm{ppm}$ with $J$ value $8.6 \mathrm{~Hz}$ attributed to H-7. On the basis of the values of coupling constant, the cis-configuration ${ }^{36}$ ) was assigned to the cycloadduct 5i. The ${ }^{1} \mathrm{H}-\mathrm{NMR}$ spectra showed that only one stereoisomer was present for all products $\mathbf{5 a}-\mathbf{p}$, indicating that the reaction 


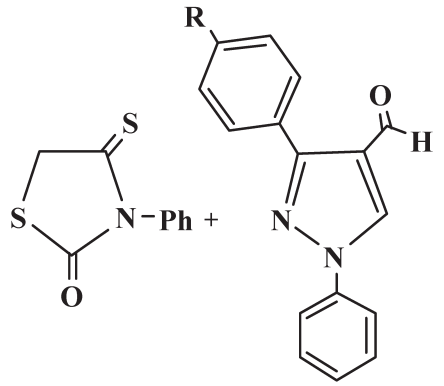

1

2a-d

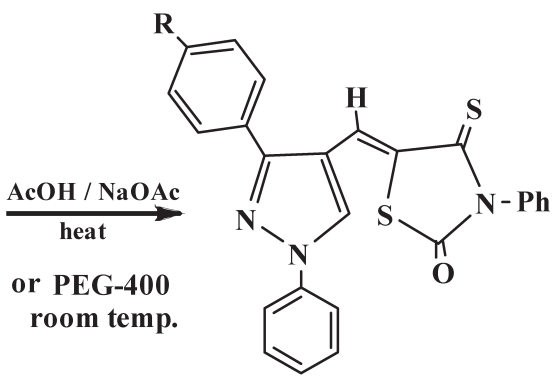

3a-d

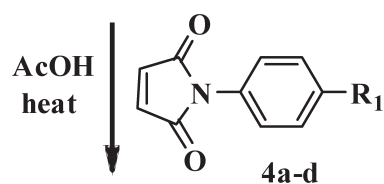

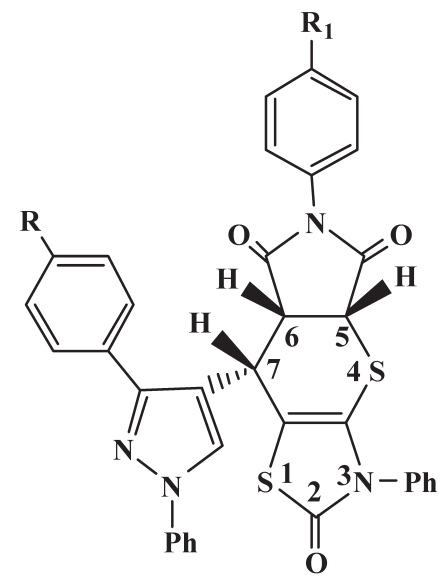

$$
\begin{aligned}
& \text { 2,3a } \mathrm{R}=\mathrm{H} \quad \text { 4a-d } \quad \text { 5a } \mathrm{R}=\mathrm{H} \quad \begin{array}{l}
\mathbf{5 a}-\mathrm{p} \\
\mathrm{R}
\end{array}
\end{aligned}
$$

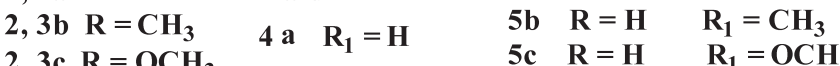

$$
\begin{aligned}
& \text { 2, 3c } \mathrm{R}=\mathrm{OCH}_{3} \\
& \mathrm{R}_{1}=\mathrm{OCH}_{3} \\
& \text { 2, 3d } \mathrm{R}=\mathrm{Cl} \\
& 4 \text { b } \mathrm{R}_{1}=\mathrm{CH}_{3} \\
& \text { 5d } \quad \mathbf{R}=\mathbf{H} \\
& \mathrm{R}_{1}=\mathbf{C l} \\
& \text { 5e } \quad \mathrm{R}=\mathrm{CH}_{3} \quad \mathrm{R}_{1}=\mathrm{H} \\
& 4 \mathrm{~d} \quad \mathrm{R}_{1}=\mathrm{Cl} \\
& \text { 5f } \mathrm{R}=\mathrm{CH}_{3} \quad \mathrm{R}_{1}=\mathrm{CH}_{3} \\
& \text { 5g } \mathrm{R}=\mathrm{CH}_{3} \quad \mathrm{R}_{1}=\mathrm{OCH}_{3} \\
& \text { 5h } \mathrm{R}=\mathrm{CH}_{3} \quad \mathrm{R}_{1}=\mathrm{Cl}
\end{aligned}
$$

\begin{tabular}{|c|c|c|}
\hline $5 \mathbf{i}$ & $\mathrm{R}=\mathrm{OCH}_{3}$ & $\mathrm{R}_{\mathbf{1}}=\mathrm{H}$ \\
\hline $5 \mathbf{j}$ & $\mathrm{R}=\mathrm{OCH}_{3}$ & $\mathrm{R}_{1}=\mathrm{CH}_{3}$ \\
\hline $5 k$ & $\mathrm{R}=\mathrm{OCH}_{3}$ & $\mathrm{R}_{1}=\mathrm{OCH}_{3}$ \\
\hline 51 & $\mathrm{R}=\mathrm{OCH}_{3}$ & $\mathrm{R}_{1}=\mathrm{Cl}$ \\
\hline $5 \mathrm{~m}$ & $\mathbf{R}=\mathbf{C l}$ & $\mathbf{R}_{1}=\mathbf{H}$ \\
\hline $5 n$ & $\mathbf{R}=\mathbf{C l}$ & $\mathrm{R}_{1}=\mathrm{CH}_{3}$ \\
\hline 50 & $\mathbf{R}=\mathbf{C l}$ & $\mathrm{R}_{1}=\mathrm{OCH}_{3}$ \\
\hline $5 p$ & $\mathbf{R}=\mathbf{C l}$ & $\mathrm{R}_{1}=\mathrm{Cl}$ \\
\hline
\end{tabular}

Chart 1. Synthesis of Compounds $\mathbf{3 a}-\mathbf{d}$ and Cycloadducts $\mathbf{5 a}-\mathbf{p}$

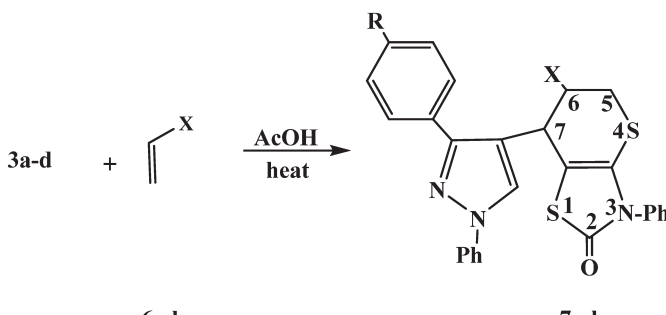

6a,b

6a $\mathrm{X}=\mathrm{CN}$

6b $\mathrm{X}=\mathrm{COOF}$

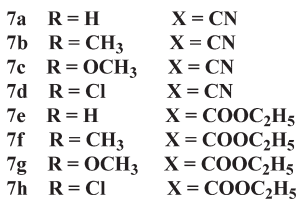

$\begin{array}{lll}7 \mathrm{~h} & \mathrm{R}=\mathrm{Cl} & \mathrm{X}=\mathrm{COOC}_{2} \mathrm{H}_{5}\end{array}$

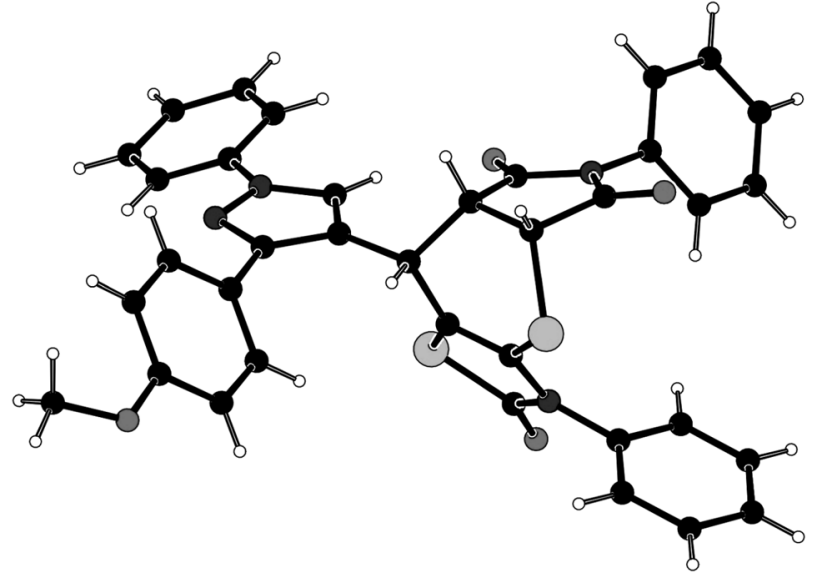

Fig. 1. X-Ray Crystallography of $\mathbf{5 i}$ 


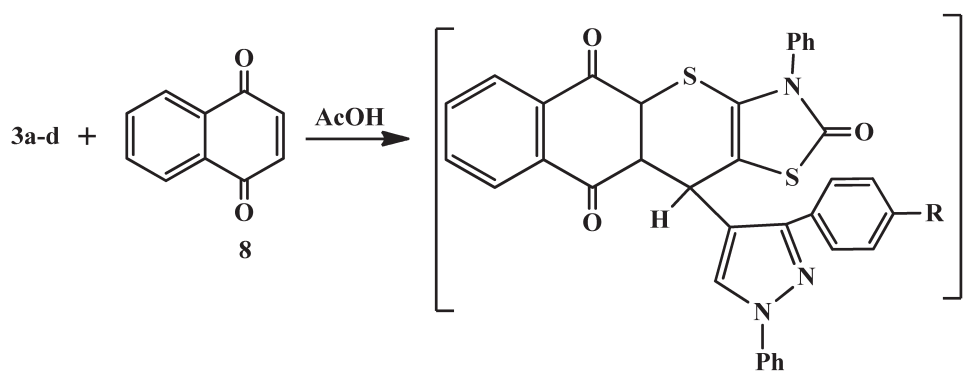<smiles>O=C1C=CC(=O)c2ccccc21</smiles>

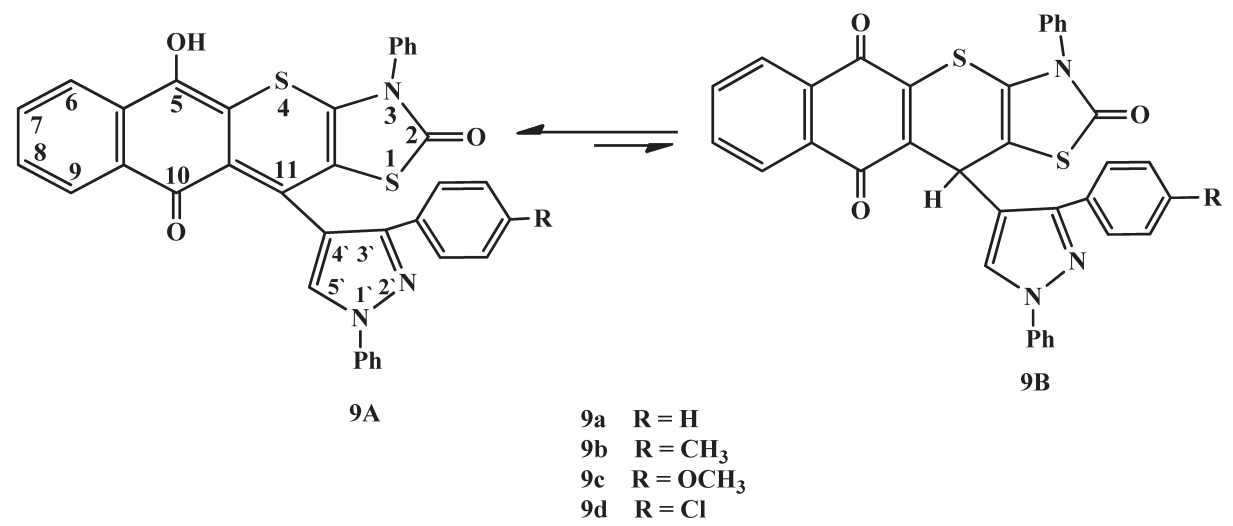

Chart 3. Synthesis of Compound 9d

is stereoselective. Also, X-ray crystallographic analysis is as a typical example for the product 5 (Fig. 1).

Similarly, the reaction of $\mathbf{3 a}-\mathbf{d}$ with acrylonitrile $\mathbf{6 a}$ and with ethyl acrylate $\mathbf{6} \mathbf{b}$ under the same reaction conditions gave cycloadducts $\mathbf{7 a}-\mathbf{h}$ as shown in Chart 2. Based on the elemental analysis and spectral data, structures $7 \mathbf{a}-\mathbf{h}$ were assigned to these adducts.

Heterocyclic compounds with naphthoquinone fragment are well known as anticancer drugs, such as doxorubicin, daunorubicin, mitoxantrone and mitomycine $C^{37,38)}$ Thus, the reaction of $\mathbf{3 a}-\mathbf{d}$ with 1,4-naphthoquinone $\mathbf{8}$ in refluxing acetic acid afforded compounds $\mathbf{9 a}-\mathbf{d}$ (Chart 3). The structure of the isolated adducts was confirmed by elemental analysis and spectral data. For example, the IR spectrum of compound 9a showed absorption bands at $v_{\max } 3347$ and $1668 \mathrm{~cm}^{-1}$ corresponding to hydroxyl and carbonyl groups, respectively. ${ }^{1} \mathrm{H}-\mathrm{NMR}$ spectrum of this isolated adduct revealed a $\mathrm{D}_{2} \mathrm{O}$ exchangeable signal at $\delta 10.0 \mathrm{ppm}$ assigned to $\mathrm{OH}$ proton at position 5. In addition to the signals for naphthoquinone moiety protons and pyrazolyl substituent in position 11 . The ${ }^{13} \mathrm{C}-\mathrm{NMR}$ spectrum with elemental analysis are in agreement with the structure 9. From the above data the synthesized novel thiopyrano[2,3- $d]$ thiazoles were assigned to structure $9 \mathbf{A}$ and ruled out the tautomer $\mathbf{9 B}$.

\section{Biological Activity}

\section{Anticancer Activity and Docking}

The main focus of biological activity studies was searching for compounds with antitumor activity. The newly synthesized compounds 3a, 5a, 5c, 5d and 7e were tested by National Cancer Institution Cairo for in vitro cell line screening. Anti-
Table 1. $\quad \mathrm{IC}_{50}$ Values of the Tested Compounds $\mathbf{3 a}, \mathbf{5 a}, \mathbf{5 c}, \mathbf{5 d}$ and $7 \mathrm{e}$ against Human Breast (MCF7) and Human Liver (HEPG2) Cell Lines

\begin{tabular}{ccc}
\hline \hline Compd No. & $\mathrm{IC}_{50}$ in $\mu \mathrm{g} / \mathrm{mL}(\mathrm{MCF} 7)$ & $\mathrm{IC}_{50}$ in $\mu \mathrm{g} / \mathrm{mL}(\mathrm{HEPG})$ \\
\hline $\mathbf{3 a}$ & 19.3 & 10.6 \\
$\mathbf{5 a}$ & 22.4 & 15.8 \\
$\mathbf{5 c}$ & 12.3 & 21.4 \\
$\mathbf{5 d}$ & 35.9 & 31.3 \\
$\mathbf{7 e}$ & 23.0 & 18.8 \\
\hline
\end{tabular}

cancer assays were performed according to US NCI protocol. The compounds were first evaluated at one dose primary anticancer assay towards approximately two cell lines. The human tumor cell lines represent two forms of cancer, human breast MCF7 and human liver HEPG2 cancer cell lines using the method of sulforhodamine B (SRB)-stain assay. In the present method, each cell line was inoculated and pre-incubated for $24-48 \mathrm{~h}$ on a microtiter plate. Different concentrations of each compound $(6,12.5,25$, and 50$)$ were used, the relation between surviving fraction and compound concentration was plotted and $\mathrm{IC}_{50}$ of each compound was showed in Table 1.

All tested compounds are showed significant anticancer activity, sensitivity of compounds against human liver cancer cell line HEPG2 exhibit $\mathrm{IC}_{50}$ ranging from 10.6 to $31.3 \mu \mathrm{g} /$ $\mathrm{mL}$. Moreover, sensitivity of compounds against human breast cancer cell line MCF7 showed $\mathrm{IC}_{50}$ range from 12.3 to $35.9 \mu \mathrm{g} /$ $\mathrm{mL}$. Compound 3a was the most active one against liver cancer cell line with $\mathrm{IC}_{50}=10.6 \mu \mathrm{g} / \mathrm{mL}$ while $\mathbf{5 c}$ was the most active one against breast cancer cell line with $\mathrm{IC}_{50}=12.3 \mu \mathrm{g} /$ 
Table 2. Docking Energy

\begin{tabular}{|c|c|c|c|c|c|}
\hline Compd No. & $\begin{array}{l}\text { Docking energy } \\
(\mathrm{kcal} / \mathrm{mol})\end{array}$ & $\begin{array}{c}\text { Atoms of ligand involved } \\
\text { in docking }\end{array}$ & $\begin{array}{l}\text { Atoms of amino acid } \\
\text { involved in docking }\end{array}$ & $\begin{array}{c}\text { Amino acids residue } \\
\text { involved in docking } \\
\text { interaction }\end{array}$ & $\begin{array}{c}\text { Length of hydrogen bond } \\
(\AA)\end{array}$ \\
\hline $3 \mathbf{a}$ & -7.17 & O 28132 & OH 3361 & TYR 224 & H-acc 3.09 \\
\hline $5 c$ & -7.85 & O 28171 & OH 3361 & TYR 224 & H-acc 2.64 \\
\hline $5 \mathbf{a}$ & -8.39 & O 28167 & OH 3357 & TYR 224 & H-acc 2.82 \\
\hline
\end{tabular}

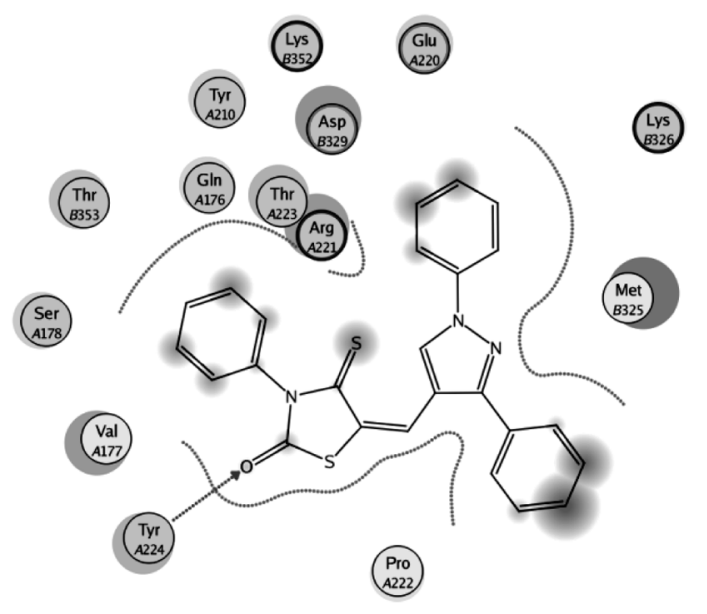

GIn

Fig. 2. Ligand Interaction of Compound 3a with Tubulin Polymerase

$\mathrm{mL}$. Compound 3a has the best reactivity toward both MCF7 and HEPG2 with $\mathrm{IC}_{50}=19.3 \mu \mathrm{g} / \mathrm{mL}$ and $10.6 \mu \mathrm{g} / \mathrm{mL}$, respectively. Compound $\mathbf{5 d}$ possessed less potent anticancer activity against both MCF7 and HEPG2. Order of antitumor activity against MCF7 was $\mathbf{5} \mathbf{c}>\mathbf{3 a}>\mathbf{5 a}>\mathbf{7 e}>\mathbf{5} \mathbf{d}$ according to their $\mathrm{IC}_{50}$, while in HEPG2 was $\mathbf{3 a}>\mathbf{5 a}>\mathbf{7 e}>\mathbf{5} \mathbf{c}>\mathbf{5 d}$. In human breast cancer cell line MCF7, using electron donating group (OMe) in the phenyl moiety as in compound $\mathbf{5 c}$ increase the reactivity rather than using electron withdrawing group $(\mathrm{Cl})$ in the phenyl moiety as in compound 5d from 12.3 to $35.9 \mu \mathrm{g} / \mathrm{mL}$. Different dienophiles are used as maleimide moieties in compounds $\mathbf{5 a}, \mathbf{5 c}$ and $\mathbf{5 d}$ or ethyl acrylate moiety as in compound 7e. The parent compound 3a showing more cytotoxic activity towards human breast cancer cell line MCF7 than in compounds 5a and 7e with phenyl maleimide moiety and ethyl acrylate moiety, respectively. The reactivity is slightly different when introducing either phenyl maleimide or ethyl acrylate moieties in the parent compound. In human liver cancer cell line HEPG2, when maleimide moiety was used as dienophile we notice that compound 5a with phenyl moiety was the most potent compound towards HEPG2 rather than adding electron donating or electron withdrawing groups into the phenyl moiety as in compounds $\mathbf{5 c}$ and $\mathbf{5 d}$. Compound $\mathbf{5 c}$ with electron donating group (OMe) in the phenyl moiety exhibits cytotoxic activity more than compound $\mathbf{5 d}$ with electron withdrawing group $(\mathrm{Cl})$ in the phenyl moiety. Using the phenyl maleimide moiety increase cytotoxic activity against liver cancer cell line more than using ethyl acrylate moiety.

Molecular Docking Study

Docking was performed against tubulin protein because it is reported as possible anticancer target of thiazolidine derivatives. $^{39)}$ Compounds $\mathbf{3 a}, \mathbf{5 c}$ and $\mathbf{5 a}$ were docked against tubu-

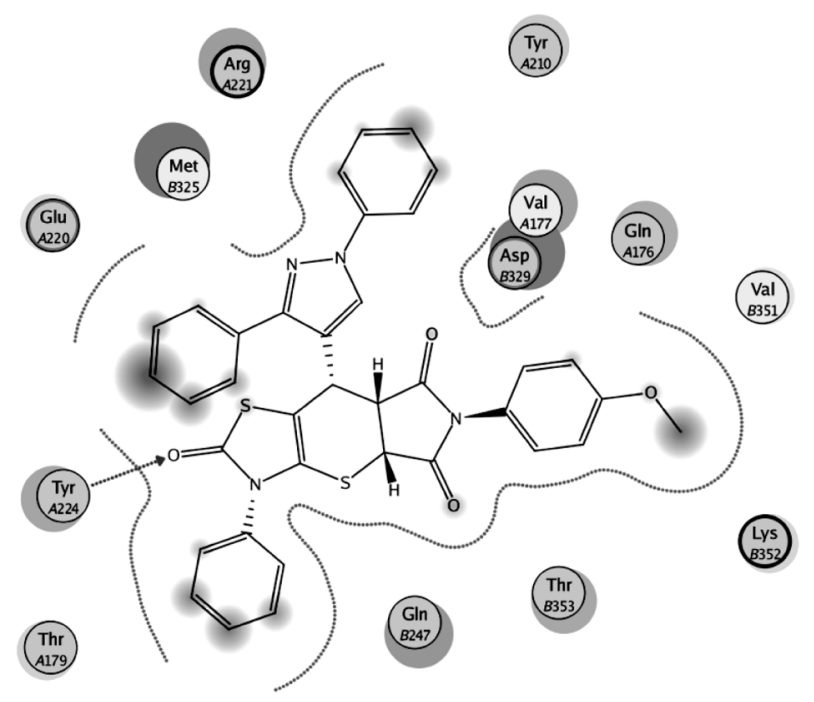

Leu

Fig. 3. Ligand Interaction of Compound $\mathbf{5 c}$ with Tubulin Polymerase

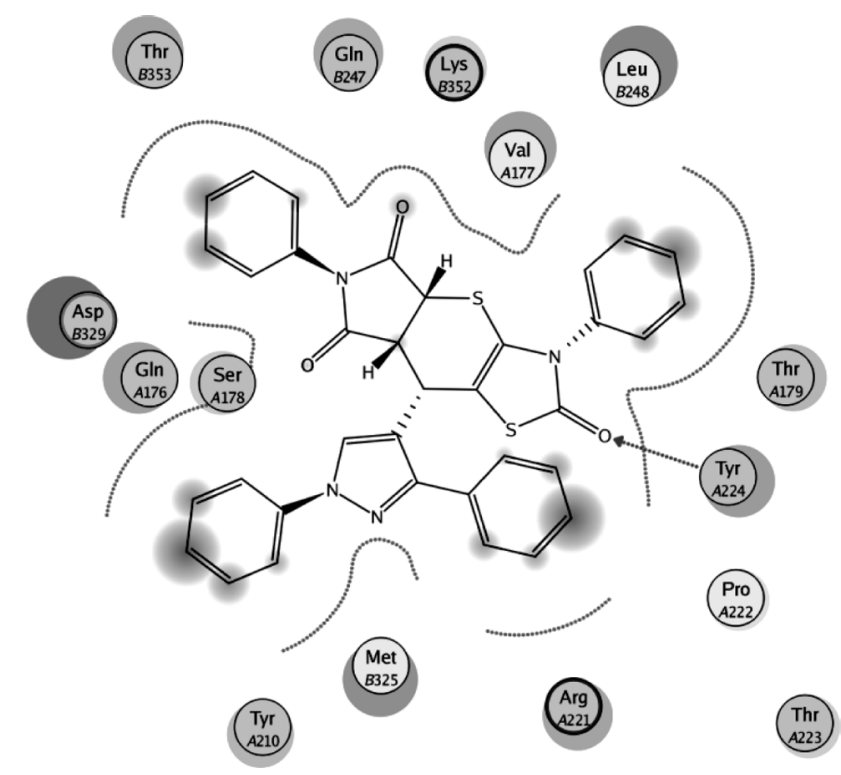

Fig. 4. Ligand Interaction of Compound 5a with Tubulin Polymerase

lin polymerase protein as an example to investigate if these compounds have a similar mechanism as tubulin polymerase inhibitors. Docking was carried out using MOE 2008.10 software, the $3 \mathrm{D}$ crystal structure of tubulin protein was obtained from protein data bank (PDB code: 1SA1) then saved as moe file, the 2D structure of docked compounds was converted into 3D structure and energy minimization was carried out then saved as mol. The docking energy was recorded for the 
selected compound interaction with tubulin protein (Table 2).

We noticed that compounds $\mathbf{3 a}, \mathbf{3} \mathbf{c}$ and $\mathbf{5 a}$ have the binding energies $-7.17,-7.85$ and $-8.39 \mathrm{kcal} / \mathrm{mol}$, respectively. They formed one hydrogen bond with TYR 224 of the active site of tubulin protein (see Figs. 2-4). We have found that all the compounds have the same interacted moiety (CO group) which formed hydrogen bond with $\mathrm{OH}$ group of the TYR 224. Binding of the synthesized compounds with the active site of tubulin protein may support the postulation that these compounds have the same mechanism of tubulin polymerase inhibitors.

\section{Conclusion}

In this paper we described the synthesis, biological activity, and structure-activity relationships of a series of thiopyrano[2,3-d]thiazolidinones. X-Ray crystal structure helped us to define the stereochemistry of the adducts.

1. Compounds $\mathbf{3 a}, \mathbf{5 a}, \mathbf{5 c}, \mathbf{5 d}$ and $\mathbf{7 e}$ showed significant anticancer activity against both liver and breast cancer cell lines.

2. The sensitivity of the latter compounds against human liver cancer cell line $\mathrm{HEPG} 2$ exhibit $\mathrm{IC}_{50}$ ranging from 10.6 to $31.3 \mu \mathrm{g} / \mathrm{mL}$. Moreover, the sensitivity of the same compounds against human breast cancer cell line MCF7 showed $\mathrm{IC}_{50}$ ranging from 12.3 to $35.9 \mu \mathrm{g} / \mathrm{mL}$.

3. The parent compound 3a has a broad spectrum antitumor activity against both liver (HEPG2) and breast (MCF7) cell lines and the reactivity decreased when using either phenyl maleimide or ethyl acrylate moieties.

4. Introducing electron withdrawing group (Chlorine atom) in the phenyl maleimide moiety as in compound $\mathbf{5 d}$ decrease the cytotoxic activity towards both liver and breast cancer cell lines.

5. The electron donating group (Methoxy group) in the phenyl maleimide moiety in compound $\mathbf{5 c}$ increase the reactivity against breast (MCF7) cell line but decrease the reactivity against liver (HEPG2) cell line rather than not using it.

6. Phenyl maleimide moiety increase the cytotoxic activity towards both liver and breast cancer cell lines more than ethyl acrylate moiety.

7. Molecular docking showed that, the compounds 3a, 5a, 5c, 5d and 7e have a similar mechanism as tubulin polymerase inhibitors.

\section{Experimental}

Chemistry All melting points were determined on an Electrothermal (9100) apparatus and are uncorrected. The IR spectra were recorded as $\mathrm{KBr}$ pellets on a PerkinElmer, Inc. 1430 spectrophotometer. The NMR spectra were recorded with a Varian Mercury VXR-300 NMR spectrometer at 300 and $75 \mathrm{MHz}$ ( ${ }^{1} \mathrm{H}$ - and ${ }^{13} \mathrm{C}-\mathrm{NMR}$ spectra, respectively) using DMSO- $d_{6}$ as solvent and results are expressed as $\delta$ values. Mass spectra were taken on a Shimadzu GCMS-QP 1000 Ex mass spectrometer at $70 \mathrm{eV}$. Elemental analyses were carried out at the Microanalyses Center at Cairo University and were performed on Vario EL III Elemental CHNS analyzer. The $\mathrm{X}$-ray crystallography was performed at Institute of Organic Chemistry of University of Zürich. Anticancer activity was performed at National Cancer Institute at Cairo University of Egypt.

The starting compounds $\mathbf{2} \mathbf{a}-\mathbf{d}$ were prepared according to lit. $^{40)}$

Preparation of 5-[(1-Phenyl-3-aryl-1H-pyrazol-4-yl)methylene]-3-phenyl-4-thioxo-2-thiazolidinone (3a-d)

General Procedure

Method A A mixture of 3-phenyl-4-thioxo-2-thiazolidinone $1(0.01 \mathrm{~mol}), 1$-phenyl-3-aryl-1H-pyrazole-4-carbaldehyde $2 \mathbf{a}-\mathbf{d}(0.01 \mathrm{~mol})$ and fused sodium acetate $(0.015 \mathrm{~mol})$ in $10 \mathrm{~mL}$ of glacial acetic acid was refluxed for $2 \mathrm{~h}$ and was left overnight at room temperature. The solid precipitated was filtered off, washed with water, dried and recrystallized from ethanoldioxane mixture.

Method B To compound $\mathbf{1}(0.01 \mathrm{~mol})$ in $5 \mathrm{~mL}$ of PEG-400, 1-phenyl-3-aryl- $1 H$-pyrazole-4-carbaldehydes $\mathbf{2 a - d}(0.01 \mathrm{~mol})$ was added. The mixture stirred at room temperature till precipitation. The mixture poured into water, filtered, washed with water, dried and recrystallized from ethanol-dioxane mixture.

5-[(1,3-Diphenyl-1H-pyrazol-4-yl)methylene]-3-phenyl-4thioxo-2-thiazolidi-none (3a)

Orange crystals; yield $=89 \% \mathbf{A}, 89 \% \mathbf{B} ; \mathrm{mp} 248^{\circ} \mathrm{C}$; IR $(\mathrm{KBr})$ $\mathrm{cm}^{-1}: 1715$ (CO); ${ }^{1} \mathrm{H}-\mathrm{NMR}\left(300 \mathrm{MHz}, \mathrm{DMSO}-d_{6}\right) \delta: 7.41-8.07$ (m, 15H, Ar), $8.21(\mathrm{~s}, 1 \mathrm{H}, \mathrm{CH}), 8.89$ (s, 1H, pyrazole-H); MS $m / z$ : $439\left(\mathrm{M}^{+}, 97.9 \%\right), 378$ (4.47\%), 346 (51.4\%), 319 (23.8\%), 287 (15.4\%), 231 (15.4\%), 189 (9.9\%), 104 (17.16\%), 77 (100\%). Anal. Calcd for $\mathrm{C}_{25} \mathrm{H}_{17} \mathrm{~N}_{3} \mathrm{OS}_{2}$ : C, 68.31; H, 3.90; N, 9.56; S, 14.59. Found: C, 68.50; H, 3.70; N, 9.30; S, 14.78 .

5 -[(1-Phenyl-3 -(4-methylphenyl)- $1 H$-pyazol-4-yl)methylene]-3-phenyl-4-thioxo-2-thiazolidinone (3b)

Orange crystals; yield $=77 \% \mathbf{A}, 80 \% \mathbf{B} ; \mathrm{mp} 251{ }^{\circ} \mathrm{C} ;{ }^{1} \mathrm{H}-\mathrm{NMR}$ $\left(300 \mathrm{MHz}, \mathrm{DMSO}-d_{6}\right) \delta: 2.38\left(\mathrm{~s}, 3 \mathrm{H}, \mathrm{CH}_{3}\right), 7.0-7.80(\mathrm{~m}, 14 \mathrm{H}$, $\mathrm{Ar}), 8.22(\mathrm{~s}, 1 \mathrm{H}, \mathrm{CH}), 8.87(\mathrm{~s}, 1 \mathrm{H}$, pyrazole-H); IR $(\mathrm{KBr})$ $\mathrm{cm}^{-1}$ : 1713 (CO); Anal. Calcd for $\mathrm{C}_{26} \mathrm{H}_{19} \mathrm{~N}_{3} \mathrm{OS}_{2}$ (453.58): C, 68.85; H, 4.22; N, 9.26; S, 14.14. Found: C, 68.68; H, 4.40; N, 9.46. S, 14.32 .

5-[(1-Phenyl-3-(4-methoxyphenyl)-1H-pyrazol-4-yl)methylene]-3-phenyl-4-thioxo-2-thiazolidinone (3c)

Orange crystals; yield $=97 \%$ A, $99 \% \mathbf{B} ; \mathrm{mp} 224^{\circ} \mathrm{C} ;{ }^{1} \mathrm{H}-\mathrm{NMR}$ $\left(300 \mathrm{MHz}, \mathrm{DMSO}-d_{6}\right) \delta: 3.84\left(\mathrm{~s}, 3 \mathrm{H}, \mathrm{OCH}_{3}\right), 7.13-8.08(\mathrm{~m}$, $14 \mathrm{H}, \mathrm{Ar}), 8.23(\mathrm{~s}, 1 \mathrm{H}, \mathrm{CH}), 8.87$ (s, 1H, pyrazole-H); IR (KBr) $\mathrm{cm}^{-1}$ : 1714 (CO); Anal. Calcd for $\mathrm{C}_{26} \mathrm{H}_{19} \mathrm{~N}_{3} \mathrm{O}_{2} \mathrm{~S}_{2}$ (469.58): C, 66.50; H, 4.08; N, 8.95; S, 13.65. Found: C, 66.32; H, 4.27; N, 9.18; S, 13.84 .

5-[(1-Phenyl-3-(4-chlorophenyl)-1H-pyrazol-4-yl)methylene]-3-phenyl-4-thioxo-2-thiazolidinone (3d)

Orange crystals; yield $=83 \% \mathbf{A}, 89 \% \mathbf{B} ; \mathrm{mp} 261{ }^{\circ} \mathrm{C} ;{ }^{1} \mathrm{H}-\mathrm{NMR}$ $\left(300 \mathrm{MHz}, \mathrm{DMSO}-d_{6}\right) \delta: 7.40-8.06(\mathrm{~m}, 14 \mathrm{H}, \mathrm{Ar}), 8.17(\mathrm{~s}, 1 \mathrm{H}$, $\mathrm{CH}), 8.89$ (s, 1H, pyrazole-H); IR (KBr) cm ${ }^{-1}: 1712$ (CO); MS $m / z$ : $474\left(\mathrm{M}^{+}, 32.3 \%\right), 380$ (40.3\%), 319 (13.2\%), 275 (15.0\%), 231 (10.6\%), 172 (7.5\%), 104 (14.3\%), 77 (100\%). Anal. Calcd for $\mathrm{C}_{25} \mathrm{H}_{16} \mathrm{ClN}_{3} \mathrm{OS}_{2}$ : C, 63.35; $\mathrm{H}, 3.40 ; \mathrm{Cl}, 7.48 ; \mathrm{N}, 8.87$; 13.53. Found: C, 63.52; H, 3.59; Cl, 7.67; N, 9.08; S, 13.71.

Preparation of 7-Aryl-3-phenyl-5,6-bis-hydroxycarbonyltetrahydro-thiopyrano- $7 \mathrm{H}[2,3-d]$ thiazol-2-one- $N$-arylimides (5a-p)

General Procedure

To each of $\mathbf{3 a}-\mathbf{d}(0.01 \mathrm{~mol})$, suspended in glacial acetic acid $(20 \mathrm{~mL})$, were added $N$-arylmaleimides $\mathbf{2 a}-\mathbf{d}(0.01 \mathrm{~mol})$. The mixture was refluxed for $30 \mathrm{~min}$, or till decolourization took place, then it was left overnight at room temperature. The solid products so obtained was filtered off, and recrystallized from ethanol-dioxane mixture. The physical constants togeth- 
er with spectral data of compounds $\mathbf{5 a}-\mathbf{p}$ are shown below.

8-(1,3-Diphenyl-1 $H$-pyrazol-4-yl)-3,6-diphenyl-7a,8dihydropyrrolo- $\left[3^{\prime}, 4^{\prime}:-5,6\right]$ thiopyrano $[2,3-d]$ thiazole2,5,7(3H,4a $H, 6 H)$-trione (5a)

White crystals; yield $=66 \% ; \mathrm{mp} 224^{\circ} \mathrm{C} ;{ }^{1} \mathrm{H}-\mathrm{NMR}(300 \mathrm{MHz}$, DMSO- $\left.d_{6}\right) \delta: 4.46(\mathrm{dd}, 1 \mathrm{H}, J=7.2,3.9 \mathrm{~Hz}, \mathrm{H}-6), 4.62(\mathrm{~d}, 1 \mathrm{H}$, $J=3.9 \mathrm{~Hz}, \mathrm{H}-5), 5.06$ (d, $1 \mathrm{H}, J=7.2 \mathrm{~Hz}, \mathrm{H}-7), 7.12-8.32(\mathrm{~m}$, $20 \mathrm{H}, \mathrm{Ar}), 8.85$ (s, 1H, pyrazole-H); IR $(\mathrm{KBr}) \mathrm{cm}^{-1}: 1718,1684$ (CO); MS m/z: $613\left(\mathrm{M}^{+}+1,57.6 \%\right), 468(86.4 \%), 377(100 \%)$, 241 (86.4\%), 200 (91.5\%), 145 (83.0\%), 95 (16.9\%), 76 (14.5\%), 59 (60.1\%). Anal. Calcd for $\mathrm{C}_{35} \mathrm{H}_{24} \mathrm{~N}_{4} \mathrm{O}_{3} \mathrm{~S}_{2}: \mathrm{C}, 68.61 ; \mathrm{H}, 3.95$; N, 9.14; S, 10.47. Found: C, 68.78; H, 3.76; N, 9.36; S, 10.65.

8 -(1,3-Diphenyl-1 H-pyrazol-4-yl)-3-phenyl- 6 - (4 methylphenyl)-7a, 8 -dihydropyrrolo[ $\left.3^{\prime}, 4^{\prime}: 5,6\right]$ thiopyrano[2,3- $\left.d\right]$ thiazole-2,5,7(3H,4aH,6H)-trione (5b)

Pale yellow crystals; yield $=70 \% ; \mathrm{mp} 218^{\circ} \mathrm{C} ;{ }^{1} \mathrm{H}-\mathrm{NMR}$ $\left(300 \mathrm{MHz}, \mathrm{DMSO}-d_{6}\right) \delta: 2.31\left(\mathrm{~s}, 3 \mathrm{H}, \mathrm{CH}_{3}\right), 4.38(\mathrm{dd}, 1 \mathrm{H}$, $J=7.8,3.9 \mathrm{~Hz}, \mathrm{H}-6), 4.53$ (d, 1H, J=3.9 Hz, H-5), 5.24 (d, $1 \mathrm{H}, J=7.8 \mathrm{~Hz}, \mathrm{H}-7), 7.06-8.41(\mathrm{~m}, 19 \mathrm{H}, \mathrm{Ar}), 8.81(\mathrm{~s}, 1 \mathrm{H}$, pyrazole-H); IR (KBr) cm $\mathrm{cm}^{-1}$ : 1714, 1684 (CO). Anal. Calcd for $\mathrm{C}_{36} \mathrm{H}_{26} \mathrm{~N}_{4} \mathrm{O}_{3} \mathrm{~S}_{2}$ (626.75): C, 68.99; H, 4.18; N, 8.94; S, 10.23. Found: C, 68.78; H, 4.36; N, 8.70; S, 10.41 .

8-(1,3-Diphenyl-1H-pyrazol-4-yl)-6-(4-methoxyphenyl)-3phenyl-7a,8-dihydropyrrolo $\left[3^{\prime}, 4^{\prime}: 5,6\right]$ thiopyrano $[2,3-d]$ thiazole-2,5,7(3H,4a $H, 6 H)$-trione $(\mathbf{5 c})$

Pale yellow crystals; yield $=86 \%$; mp $220^{\circ} \mathrm{C}$; ${ }^{1} \mathrm{H}-\mathrm{NMR}$ $\left(300 \mathrm{MHz}, \mathrm{DMSO}-d_{6}\right) \delta: 3.78\left(\mathrm{~s}, 3 \mathrm{H}, \mathrm{OCH}_{3}\right), 4.34(\mathrm{dd}, 1 \mathrm{H}$, $J=8.7,3.6 \mathrm{~Hz}, \mathrm{H}-6), 4.50$ (d, $1 \mathrm{H}, J=3.6 \mathrm{~Hz}, \mathrm{H}-5), 4.94$ (d, $1 \mathrm{H}, J=8.7 \mathrm{~Hz}, \mathrm{H}-7), 6.92-7.81(\mathrm{~m}, 19 \mathrm{H}, \mathrm{Ar}), 8.75(\mathrm{~s}, 1 \mathrm{H}$, pyrazole-H); IR (KBr) cm $\mathrm{cm}^{-1}$ : 1718, 1685 (CO). Anal. Calcd for $\mathrm{C}_{36} \mathrm{H}_{26} \mathrm{~N}_{4} \mathrm{O}_{4} \mathrm{~S}_{2}$ (642.75): C, 67.27; $\mathrm{H}, 4.08 ; \mathrm{N}, 8.72 ; \mathrm{S}, 9.98$. Found: C, 67.44; H, 4.26; N, 8.48; S, 9.80.

6-(4-Chlorophenyl)-8-(1,3-diphenyl-1H-pyrazol-4-yl)-3phenyl-7a,8-di-hydropyrrolo $\left[3^{\prime}, 4^{\prime}: 5,6\right]$ thiopyrano $[2,3-d]$ thiazole-2,5,7(3H,4aH,6H)-trione (5d)

Pale yellow crystals; yield $=80 \% ; \mathrm{mp} 225^{\circ} \mathrm{C}$; ${ }^{1} \mathrm{H}-\mathrm{NMR}$ $\left(300 \mathrm{MHz}, \mathrm{DMSO}-d_{6}\right) \delta: 4.36(\mathrm{dd}, 1 \mathrm{H}, J=8.7,4.2 \mathrm{~Hz}, \mathrm{H}-6)$, $4.51(\mathrm{~d}, 1 \mathrm{H}, J=4.2 \mathrm{~Hz}, \mathrm{H}-5), 4.96(\mathrm{~d}, 1 \mathrm{H}, J=8.7 \mathrm{~Hz}, \mathrm{H}-7)$, 7.10-7.87 (m, 19H, Ar), $8.75(\mathrm{~s}, 1 \mathrm{H}$, pyrazole-H); IR $(\mathrm{KBr})$ $\mathrm{cm}^{-1}$ : 1712, 1687 (CO). Anal. Calcd for $\mathrm{C}_{35} \mathrm{H}_{23} \mathrm{ClN}_{4} \mathrm{O}_{3} \mathrm{~S}_{2}$ (647.16): C, 64.96; H, 3.58; Cl, 5.48; N, 8.66; S, 9.91. Found: C, 64.79; H, 3.76; Cl, 5.65; N, 8.90; S, 9.72 .

3,6-Diphenyl-8-(1-phenyl-3-(4-methylphenyl)- $1 H$ pyrazol-4-yl)-7a,8-dihydropyrrolo[3', $\left.4^{\prime}: 5,6\right]$ thiopyrano[2,3-d]thiazole-2,5,7(3H,4a $H, 6 H)$-trione $(\mathbf{5 e})$

Pale yellow crystals; yield $=80 \%$; mp $173^{\circ} \mathrm{C}$; ${ }^{1} \mathrm{H}-\mathrm{NMR}$ $\left(300 \mathrm{MHz}, \mathrm{DMSO}-d_{6}\right) \delta: 2.36\left(\mathrm{~s}, 3 \mathrm{H}, \mathrm{CH}_{3}\right), 4.33(\mathrm{dd}, 1 \mathrm{H}$, $J=8.3,3.9 \mathrm{~Hz}, \mathrm{H}-6), 4.48$ (d, $1 \mathrm{H}, J=3.9 \mathrm{~Hz}, \mathrm{H}-5), 4.94$ (d, $1 \mathrm{H}, J=8.3 \mathrm{~Hz}, \mathrm{H}-7), 7.07-8.22$ (m, 19H, Ar), 8.71 (s, 1H, pyrazole-H); IR (KBr) cm $\mathrm{cm}^{-1}$ : 1720, 1695 (CO). Anal. Calcd for $\mathrm{C}_{36} \mathrm{H}_{26} \mathrm{~N}_{4} \mathrm{O}_{3} \mathrm{~S}_{2}$ (626.75): C, 68.99; H, 4.18; N, 8.94; S, 10.23. Found: C, 69.18; H, 4.0; N, 9.16; S, 10.05.

3-Phenyl-8-(1-phenyl-3-(4-methylphenyl)-1H-pyrazol-4yl)-6-(4-methylphenyl)-7a,8-dihydropyrolo $\left[3^{\prime}, 4^{\prime}: 5,6\right]-$ thiopyrano[2,3- $d]$ thiazole-2,5,7-(3H,4a $H, 6 H)$-trione (5f)

Pale yellow crystals; yield $=64 \% ; \mathrm{mp} 165^{\circ} \mathrm{C} ;{ }^{1} \mathrm{H}-\mathrm{NMR}$ $\left(300 \mathrm{MHz}, \mathrm{DMSO}-d_{6}\right) \delta: 2.38\left(\mathrm{~s}, 6 \mathrm{H}, 2 \mathrm{CH}_{3}\right), 4.34(\mathrm{dd}, 1 \mathrm{H}$, $J=8.4,4.2 \mathrm{~Hz}, \mathrm{H}-6), 4.81$ (d, $1 \mathrm{H}, J=4.2 \mathrm{~Hz}, \mathrm{H}-5), 5.70$ (d, $1 \mathrm{H}, J=8.4 \mathrm{~Hz}, \mathrm{H}-7), 7.10-7.80(\mathrm{~m}, 18 \mathrm{H}, \mathrm{Ar}), 8.40(\mathrm{~s}, 1 \mathrm{H}$, pyrazole-H); IR (KBr) cm $\mathrm{cm}^{-1}$ : 1720, 1682 (CO). Anal. Calcd for $\mathrm{C}_{37} \mathrm{H}_{28} \mathrm{~N}_{4} \mathrm{O}_{3} \mathrm{~S}_{2}$ (640.78): C, 69.35; H, 4.40; N, 8.74; S, 10.01.
Found: C, 69.17; H, 4.20; N, 8.98; S, 10.20.

6 - (4 - Methoxy phenyl)-3 - phenyl-8 - (1-phenyl-3 - (4 methylphenyl)-1H-pyrazol-4-yl)-7a,8-dihydropyrrolo[3',4':5,6]thiopyrano[2,3-d]thiazole-2,5,7-(3H,4a $H,-6 H)$-trione $(\mathbf{5 g})$

Pale yellow crystals; yield $=65 \%$; mp $185^{\circ} \mathrm{C}$; ${ }^{1} \mathrm{H}-\mathrm{NMR}$ $\left(300 \mathrm{MHz}, \mathrm{DMSO}-d_{6}\right) \delta: 2.37\left(\mathrm{~s}, 3 \mathrm{H}, \mathrm{CH}_{3}\right), 3.76\left(\mathrm{~s}, 3 \mathrm{H}, \mathrm{OCH}_{3}\right)$, 4.35 (dd, $1 \mathrm{H}, J=8.7,3.9 \mathrm{~Hz}, \mathrm{H}-6), 4.52$ (d, $1 \mathrm{H}, J=3.9 \mathrm{~Hz}, \mathrm{H}-5)$, 4.94 (d, 1H, J=8.7 Hz, H-7), 7.06-7.87 (m, 18H, Ar), 8.71 (s, $1 \mathrm{H}$, pyrazole-H); IR (KBr) cm $\mathrm{cm}^{-1}: 1714,1690$ (CO). Anal. Calcd for $\mathrm{C}_{37} \mathrm{H}_{28} \mathrm{~N}_{4} \mathrm{O}_{4} \mathrm{~S}_{2}$ (656.77): C, 67.67; H, 4.30; N, 8.53; S, 9.76. Found: C, 67.84; H, 4.48; N, 8.29; S, 9.59.

6 - (4 - Chlorophenyl)-3 - phenyl- 8 - (1 - phenyl-3 - (4 methylphenyl)-1H-pyrazol-4-yl)-7a,8-dihydropyrrolo[3',4':5,6]thiopyrano[2,3-d] thiazole-2,5,7(3H,4aH,6H)-trione (5h)

Yellow crystals; yield $=68 \%$; $\mathrm{mp} \quad 206^{\circ} \mathrm{C} ; \quad{ }^{1} \mathrm{H}-\mathrm{NMR}$ $\left(300 \mathrm{MHz}, \mathrm{DMSO}-d_{6}\right) \delta: 2.38\left(\mathrm{~s}, 3 \mathrm{H}, \mathrm{CH}_{3}\right), 4.34(\mathrm{dd}, 1 \mathrm{H}$, $J=8.4,3.9 \mathrm{~Hz}, \mathrm{H}-6), 4.49$ (d, 1H, $J=3.9 \mathrm{~Hz}, \mathrm{H}-5), 4.95$ (d, 1H, $J=8.4 \mathrm{~Hz}, \mathrm{H}-7$ ), 7.08-8.23 (m, 18H, Ar), 8.73 (s, 1H, pyrazole$\mathrm{H})$; IR (KBr) $\mathrm{cm}^{-1}$ : 1712, $1686(\mathrm{CO}) ; \mathrm{MS} \mathrm{m} / z$ : $662\left(\mathrm{M}^{+}+1\right.$, 12.7\%), 505 (16.6\%), 434 (17.2\%), 301 (22.4\%), $221(26.0 \%)$, 167 (15.7\%), 111 (28.6\%), 83 (67.8\%), 57 (100\%). Anal. Calcd for $\mathrm{C}_{36} \mathrm{H}_{25} \mathrm{ClN}_{4} \mathrm{O}_{3} \mathrm{~S}_{2}: \mathrm{C}, 65.40 ; \mathrm{H}, 3.81 ; \mathrm{Cl}, 5.36 ; \mathrm{N}, 8.47 ; \mathrm{S}$, 9.70. Found: C, 65.58; H, 3.61; Cl, 5.55; N, 8.70; S, 9.87.

8-(3-(4-Methoxyphenyl)-1-phenyl-1H-pyrazol-4-yl)-3,6diphenyl-7a, 8-dihydropyrrolo $\left[3^{\prime}, 4^{\prime}: 5,6\right]$ thiopyrano $[2,3-d]$ thiazole-2,5,7(3H,4aH,6H)-trione $(\mathbf{5 i})$

Pale yellow crystals; yield $=60 \%$; mp $219^{\circ} \mathrm{C}$; ${ }^{1} \mathrm{H}-\mathrm{NMR}$ $(300 \mathrm{MHz}, \mathrm{DMSO}) \delta: 3.76\left(\mathrm{~s}, 3 \mathrm{H}, \mathrm{OCH}_{3}\right), 4.35(\mathrm{dd}, 1 \mathrm{H}$, $J=8.6,3.6 \mathrm{~Hz}, \mathrm{H}-6), 4.52$ (d, $1 \mathrm{H}, J=3.6 \mathrm{~Hz}, \mathrm{H}-5), 4.95$ (d, $1 \mathrm{H}, J=8.6 \mathrm{~Hz}, \mathrm{H}-7), 7.08-7.82(\mathrm{~m}, 19 \mathrm{H}, \mathrm{Ar}), 8.78(\mathrm{~s}, 1 \mathrm{H}$, pyrazole-H); IR (KBr, $v \mathrm{~cm}^{-1}$ ): 1718, 1689 (CO). Anal. Calcd for $\mathrm{C}_{36} \mathrm{H}_{26} \mathrm{~N}_{4} \mathrm{O}_{4} \mathrm{~S}_{2}$ (642.75): C, 67.27; H, 4.08; N, 8.72; S, 9.98. Found: C, 67.46; H, 4.26; N, 8.49; S, 9.80.

8-(3-(4-Methoxyphenyl)-1-phenyl-1H-pyrazol-4-yl)-3phenyl-6-(methylphenyl)-7a,8-dihydropyrolo[3', $\left.4^{\prime}: 5,6\right]-$ thiopyrano[2,3-d] thiazole $2,5,7(3 H, 4 \mathrm{a} H,-6 H)$-trione $(\mathbf{5 j})$

White crystals; yield $=77 \%$; mp $214^{\circ} \mathrm{C}$; IR $(\mathrm{KBr}) \mathrm{cm}^{-1}$ : 1715, 1687 (CO); ${ }^{1} \mathrm{H}-\mathrm{NMR}\left(300 \mathrm{MHz}, \mathrm{DMSO}-d_{6}\right) \delta: 2.38$ (s, $\left.3 \mathrm{H}, \mathrm{CH}_{3}\right), 3.81\left(\mathrm{~s}, 3 \mathrm{H}, \mathrm{OCH}_{3}\right), 4.34(\mathrm{dd}, 1 \mathrm{H}, J=8.7,3.9 \mathrm{~Hz}$, H-6), 4.52 (d, 1H, J=3.9 Hz, H-5), 4.96 (d, 1H, $J=8.7 \mathrm{~Hz}, \mathrm{H}-7)$, 6.89-7.85 (m, 18H, Ar), 8.72 (s, 1H, pyrazole-H). Anal. Calcd for $\mathrm{C}_{37} \mathrm{H}_{28} \mathrm{~N}_{4} \mathrm{O}_{4} \mathrm{~S}_{2}$ (656.77): C, 67.67; H, 4.30; N, 8.53; S, 9.76. Found: C, 67.84; H, 4.13; N, 8.77; S, 9.95.

6-(4-Methoxyphenyl)-8-(3-(4-methoxyphenyl)-1-phenyl-1 Hpyrazol-4-yl)-3-phenyl-7a, 8 -dihydropyrrolo[ $\left[3^{\prime}, 4^{\prime}: 5,6\right]$ thiopyrano[2,3- $d$ ] thiazole-2,5,7(3H,4a $H,-6 H)$-trione $(\mathbf{5 k})$

Brownish yellow crystals; yield $=65 \%$; mp $210^{\circ} \mathrm{C}$; ${ }^{1} \mathrm{H}-\mathrm{NMR}$ $\left(300 \mathrm{MHz}, \mathrm{DMSO}-d_{6}\right) \delta: 3.72\left(\mathrm{~s}, 3 \mathrm{H}, \mathrm{OCH}_{3}\right), 3.76(\mathrm{~s}, 3 \mathrm{H}$, $\left.\mathrm{OCH}_{3}\right), 4.38(\mathrm{dd}, 1 \mathrm{H}, J=8.1,3.6 \mathrm{~Hz}, \mathrm{H}-6), 4.55$ (d, $1 \mathrm{H}$, $J=3.6 \mathrm{~Hz}, \mathrm{H}-5), 4.94$ (d, 1H, $J=8.1 \mathrm{~Hz}, \mathrm{H}-7), 7.05-7.87$ (m, $18 \mathrm{H}, \mathrm{Ar}), 8.78$ (s, 1H, pyrazole-H); IR (KBr) $\mathrm{cm}^{-1}: 1716,1689$ (CO). Anal. Calcd for $\mathrm{C}_{37} \mathrm{H}_{28} \mathrm{~N}_{4} \mathrm{O}_{5} \mathrm{~S}_{2}$ (672.77): C, 66.06; $\mathrm{H}$, 4.20; N, 8.33; S, 9.53. Found: C, 66.23; H, 4.37; N, 8.10; S, 9.70 .

6-(4-Chlorophenyl)-8-(3-(4-methoxyphenyl)-1-phenyl-1Hpyrazol-4-yl)-3-phenyl-7a, 8-dihydropyrrolo[3', $\left.4^{\prime}: 5,6\right]$ thiopyrano[2,3- $d$ ] thiazole-2,5,7-(3H,4a $H,-6 H)$-trione (5I)

White crystals; yield $=60 \%$; mp $218^{\circ} \mathrm{C}$; ${ }^{1} \mathrm{H}-\mathrm{NMR}(300 \mathrm{MHz}$, DMSO- $\left.d_{6}\right) \delta: 3.78\left(\mathrm{~s}, 3 \mathrm{H}, \mathrm{OCH}_{3}\right), 4.36(\mathrm{dd}, 1 \mathrm{H}, J=8.7,4.2 \mathrm{~Hz}$, H-6), 4.51 (d, 1H, J=4.2 Hz, H-5), 4.96 (d, $1 \mathrm{H}, J=8.7 \mathrm{~Hz}$, H-7), 6.87-7.88 (m, 18H, Ar), 8.75 (s, 1H, pyrazole-H); IR 
(KBr) cm ${ }^{-1}: 1714,1691$ (CO). Anal. Calcd for $\mathrm{C}_{36} \mathrm{H}_{25} \mathrm{ClN}_{4} \mathrm{O}_{4} \mathrm{~S}_{2}$ (677.19): C, 63.85; H, 3.72; Cl, 5.23; N, 8.27; S, 9.47. Found: C, 63.67; H, 3.90; Cl, 5.05; N, 8.50; S, 9.30.

8-(3-(4-Chlorophenyl)-1-phenyl-1H-pyrazol-4-yl)-3,6diphenyl-7a,8-dihydro-pyrrolo[ $\left[3^{\prime}, 4^{\prime}: 5,6\right]$ thiopyrano[2,3-d]thiazole-2,5,7(3H,4a $H, 6 H)$-trione $(\mathbf{5 m})$

Pale yellow crystals; yield $=85 \%$; mp $178^{\circ} \mathrm{C}$; ${ }^{1} \mathrm{H}-\mathrm{NMR}$ (300 MHz, DMSO): $\delta=4.38(\mathrm{dd}, 1 \mathrm{H}, J=8.6,3.9 \mathrm{~Hz}, \mathrm{H}-6), 4.41$ (d, $1 \mathrm{H}, J=3.9 \mathrm{~Hz}, \mathrm{H}-5), 4.83$ (d, $1 \mathrm{H}, J=8.6 \mathrm{~Hz}, \mathrm{H}-7), 7.15-7.86$ (m, 19H, Ar), 8.74 (s, 1H, pyrazole-H); IR (KBr, $\left.v \mathrm{~cm}^{-1}\right)$ : 1718, 1689 (CO). Anal. Calcd for $\mathrm{C}_{35} \mathrm{H}_{23} \mathrm{ClN}_{4} \mathrm{O}_{3} \mathrm{~S}_{2}$ (647.16): C, 64.96; H, 3.58; Cl, 5.48; N, 8.66; S, 9.91. Found: C, 64.78; H, 3.39; $\mathrm{Cl}, 5.30 ; \mathrm{N}, 8.90 ; \mathrm{S}, 9.72$.

8-(3-(4-Chlorophenyl)-1-phenyl-1H-pyrazol-4-yl)-3-phenyl6-(4-methylphenyl)-3,4a,7a,8-tetrahydropyrrolo[3',4':5,6]thiopyrano[2,3- $d$ ] thiazole-2,5,7(3H,4a $\mathrm{H}, 6 \mathrm{H})$-trione (5n)

Pale yellow crystals; yield $=75 \%$; mp $200^{\circ} \mathrm{C}$; ${ }^{1} \mathrm{H}-\mathrm{NMR}$ $\left(300 \mathrm{MHz}, \mathrm{DMSO}-d_{6}\right) \delta: 2.33\left(\mathrm{~s}, 3 \mathrm{H}, \mathrm{CH}_{3}\right), 4.43(\mathrm{dd}, 1 \mathrm{H}$, $J=7.2,3.9 \mathrm{~Hz}, \mathrm{H}-6), 4.51$ (d, $1 \mathrm{H}, J=3.9 \mathrm{~Hz}, \mathrm{H}-5), 5.26$ (d, $1 \mathrm{H}, J=7.2 \mathrm{~Hz}, \mathrm{H}-7), 7.13-8.42(\mathrm{~m}, 18 \mathrm{H}, \mathrm{Ar}), 8.81(\mathrm{~s}, 1 \mathrm{H}$, pyrazole-H); IR (KBr) cm $\mathrm{cm}^{-1}$ : 1714, 1687 (CO). Anal. Calcd for $\mathrm{C}_{36} \mathrm{H}_{25} \mathrm{ClN}_{4} \mathrm{O}_{3} \mathrm{~S}_{2}$ (661.19): C, 65.40; H, 3.81; Cl, 5.36; N, 8.47; S, 9.70. Found: C, 65.20; H, 3.98; Cl,5.54; N, 8.24; S, 9.88.

8-(3-(4-Chlorophenyl)-1-phenyl-1H-pyrazol-4-yl)-6-(4methoxyphenyl)-3-phenyl-3,4a,7a,8-tetrahydropyrrolo[ $\left[3^{\prime}, 4^{\prime}: 5,6\right]-$ thiopyrano[2,3- $d]$-thiazole-2,5,7(3H,4a $H, 6 H)$-trione (5o)

White crystals; yield $=64 \%$; mp $192^{\circ} \mathrm{C} ;{ }^{1} \mathrm{H}-\mathrm{NMR}(300 \mathrm{MHz}$, DMSO- $\left.d_{6}\right) \delta: 3.77\left(\mathrm{~s}, 3 \mathrm{H}, \mathrm{OCH}_{3}\right), 4.33(\mathrm{dd}, 1 \mathrm{H}, J=8.7,3.6 \mathrm{~Hz}$, H-6), 4.5 (d, 1H, J=3.6 Hz, H-5), 4.92 (d, 1H, $J=8.7 \mathrm{~Hz}, \mathrm{H}-7$ ), 6.89-7.87 (m, 18H, Ar), $8.76(\mathrm{~s}, 1 \mathrm{H}$, pyrazole-H); IR $(\mathrm{KBr})$ $\mathrm{cm}^{-1}: 1713,1688(\mathrm{CO}) ; \mathrm{MS} \mathrm{m} / z: 677\left(\mathrm{M}^{+}, 62 \%\right), 602(80.3 \%)$, $517(79.5 \%), 463(86.0 \%), 325(90.2 \%), 236(67.2 \%), 172$ (63.9\%), 122 (47.5\%), $72(100 \%), 52$ (77.0\%). Anal. Calcd for $\mathrm{C}_{36} \mathrm{H}_{25} \mathrm{ClN}_{4} \mathrm{O}_{4} \mathrm{~S}_{2}: \mathrm{C}, 63.85 ; \mathrm{H}, 3.72 ; \mathrm{Cl}, 5.23 ; \mathrm{N}, 8.27 ; \mathrm{S}, 9.47$. Found: C, 64.04; H, 3.52; Cl, 5.05; N, 8.04; S, 9.65.

6-(4-Chlorophenyl)-8-(3-(4-chlorophenyl)-1-phenyl-1 $H$ pyrazol-4-yl)-3-phenyl-3,4a,7a,8-tetrahydropyrrolo[3', 4':5,6]thiopyrano[2,3- $d]$ thiazole-2,5,7(3H,4a $\mathrm{H}, 6 \mathrm{H})$-trione (5p)

Pale yellow crystals; yield $=68 \%$; mp $202^{\circ} \mathrm{C}$; ${ }^{1} \mathrm{H}-\mathrm{NMR}$ $\left(300 \mathrm{MHz}, \mathrm{DMSO}-d_{6}\right) \delta: 4.36(\mathrm{dd}, 1 \mathrm{H}, J=8.7,3.9 \mathrm{~Hz}, \mathrm{H}-6)$, $4.52(\mathrm{~d}, 1 \mathrm{H}, J=3.9 \mathrm{~Hz}, \mathrm{H}-5), 4.97(\mathrm{~d}, 1 \mathrm{H}, J=8.7 \mathrm{~Hz}, \mathrm{H}-7)$, 7.08-7.87 (m, 18H, Ar), 8.75 (s, 1H, pyrazole-H); IR (KBr) $\mathrm{cm}^{-1}$ : 1713, 1689 (CO). Anal. Calcd for $\mathrm{C}_{35} \mathrm{H}_{22} \mathrm{Cl}_{2} \mathrm{~N}_{4} \mathrm{O}_{3} \mathrm{~S}_{2}$ (681.61): C, 61.68; H, 3.25; Cl, 10.40; N, 8.22; S, 9.41. Found: C, 61.85; H, 3.07; Cl, 10.21; N, 8.48; S, 9.60.

\section{Preparation of Cycloadducts $7 \mathbf{a}-\mathbf{h}$}

General Procedure

To each of 3a-d $(0.01 \mathrm{~mol})$, suspended in glacial acetic acid $(20 \mathrm{~mL})$, was added acrylonitrile $\mathbf{6 a}$ or ethyl acrylate $\mathbf{6 b}$ $(0.01 \mathrm{~mol})$. The mixture was refluxed for $30 \mathrm{~min}$, or till decolourization took place, then it was left overnight at room temperature. The solid products so formed was filtered off, and recrystallized from ethanol-dioxane mixture.

7-(1,3-Diphenyl-1H-pyrazol-4-yl)-2-oxo-3-phenyl-3,5,6,7tetrahydro-2 $H$-thiopyrano[2,3- $d$ ] thiazole-6-carbonitrile (7a)

Pale yellow crystals; yield $=66 \%$ mp $246^{\circ} \mathrm{C} ;{ }^{1} \mathrm{H}-\mathrm{NMR}$ $\left(300 \mathrm{MHz}, \mathrm{DMSO}-d_{6}\right) \delta: 3.44-3.53(\mathrm{~m}, 2 \mathrm{H}, \mathrm{H}-5), 4.02(\mathrm{t}, 1 \mathrm{H}$, $J=5.4 \mathrm{~Hz}, \mathrm{H}-6), 4.55(\mathrm{~d}, 1 \mathrm{H}, J=5.4 \mathrm{~Hz}, \mathrm{H}-7), 7.30-7.98(\mathrm{~m}$, $15 \mathrm{H}, \mathrm{Ar}), 8.71(\mathrm{~s}, 1 \mathrm{H}$, pyrazole-H); IR ( $\mathrm{KBr}) \mathrm{cm}^{-1}: 2240(\mathrm{CN})$, 1651 (CO). Anal. Calcd for $\mathrm{C}_{28} \mathrm{H}_{20} \mathrm{~N}_{4} \mathrm{OS}_{2}$ (492.62): C, 68.27; $\mathrm{H}$, 4.09; N, 11.37; S, 13.02. Found: C, 68.44; H, 4.29; N, 11.13; S,
13.20 .

7-[1-Phenyl-3-(4-methylphenyl)-1H-pyrazol-4-yl]-2-oxo-3phenyl-3,5,6,7-tetrahydro-2 $H$-thiopyrano[2,3-d] thiazole-6carbonitrile $\mathbf{( 7 b )}$

Pale yellow crystals; yield $=58 \%$; mp $220^{\circ} \mathrm{C}$; ${ }^{1} \mathrm{H}-\mathrm{NMR}$ $\left(300 \mathrm{MHz}, \mathrm{DMSO}-d_{6}\right) \delta: 2.38\left(\mathrm{~s}, 3 \mathrm{H}, \mathrm{CH}_{3}\right), 3.45-3.57(\mathrm{~m}, 2 \mathrm{H}$, $\mathrm{H}-5), 4.01$ (t, 1H, $J=5.4 \mathrm{~Hz}, \mathrm{H}-6), 4.60$ (d, $1 \mathrm{H}, J=5.4 \mathrm{~Hz}, \mathrm{H}-7)$, 7.31-7.96 (m, 14H, Ar), $8.70(\mathrm{~s}, 1 \mathrm{H}$, pyrazole-H); IR $(\mathrm{KBr})$ $\mathrm{cm}^{-1}: 2238(\mathrm{CN}), 1661$ (CO). MS m/z: $507\left(\mathrm{M}^{+}, 3.6 \%\right), 453$ (48.3\%), 360 (39.1\%), 319 (8.8\%), 258 (5.2\%), 229 (3.9\%), 154 (5.4\%), 103 (8.9\%), 77 (100\%), 51 (43.5\%). Anal. Calcd for $\mathrm{C}_{29} \mathrm{H}_{22} \mathrm{~N}_{4} \mathrm{OS}_{2}$ : C, 68.75; H, 4.38; N, 11.06; S, 12.66. Found: C, 68.58; H, 4.20; N, 11.29; S, 12.84 .

7-[1-Phenyl-3-(4-methoxyphenyl)-1H-pyrazol-4-yl]-2-oxo-3phenyl-3,5,6,7-tetrahydro-2 $H$-thiopyrano[2,3-d] thiazole-6carbonitrile (7c)

White crystals;yield $=88 \%$; mp $210^{\circ} \mathrm{C} ;{ }^{1} \mathrm{H}-\mathrm{NMR}(300 \mathrm{MHz}$, DMSO- $\left.d_{6}\right) \delta: 3.59-3.68(\mathrm{~m}, 2 \mathrm{H}, \mathrm{H}-5), 3.78\left(\mathrm{~s}, 3 \mathrm{H}, \mathrm{OCH}_{3}\right), 4.05$ (t, $1 \mathrm{H}, J=5.7 \mathrm{~Hz}, \mathrm{H}-6), 4.64$ (d, $1 \mathrm{H}, J=5.7 \mathrm{~Hz}, \mathrm{H}-7), 7.38-8.06$ (m, 14H, Ar), 8.73 (s, 1H, pyrazole-H); IR (KBr) $\mathrm{cm}^{-1}: 2240$ (CN), 1658 (CO). Anal. Calcd for $\mathrm{C}_{29} \mathrm{H}_{22} \mathrm{~N}_{4} \mathrm{O}_{2} \mathrm{~S}_{2}$ (522.64): C, 66.65; H, 4.24; N, 10.72; S, 12.27. Found: C, 66.81; H, 4.06; N, $10.95 ; \mathrm{S}, 12.47$.

7-[1-Phenyl-3-(4-chlorophenyl)-1H-pyrazol-4-yl]-2-oxo-3phenyl-3,5,6,7-tetrahydro- $2 H$-thiopyrano[2,3-d] thiazole-6carbonitrile (7d)

Yellow crystals; yield $=65 \%$; mp $236^{\circ} \mathrm{C} ; \quad{ }^{1} \mathrm{H}-\mathrm{NMR}$ $\left(300 \mathrm{MHz}, \mathrm{DMSO}-d_{6}\right) \delta: 3.46-3.57(\mathrm{~m}, 2 \mathrm{H}, \mathrm{H}-5), 4.0(\mathrm{t}, 1 \mathrm{H}$, $J=5.4 \mathrm{~Hz}, \mathrm{H}-6), 4.64$ (d, 1H, $J=5.4 \mathrm{~Hz}, \mathrm{H}-7), 7.37-8.02(\mathrm{~m}$, $14 \mathrm{H}, \mathrm{Ar}), 8.73$ (s, 1H, pyrazole-H); IR (KBr) cm ${ }^{-1}: 2240(\mathrm{CN})$, 1659 (CO); MS m/z: $528\left(\mathrm{M}^{+}+1,11.2 \%\right), 473(100 \%), 380$ (52.5\%), 231 (12.5\%), 190 (2.4\%), 145 (3.3\%), $111(4.2 \%), 91$ (3.9\%), 77 (89.8\%). Anal. Calcd for $\mathrm{C}_{28} \mathrm{H}_{19} \mathrm{ClN}_{4} \mathrm{OS}_{2}$ : C, 63.81; H, 3.63; Cl, 6.73; N, 10.63; S, 12.17. Found: C, 63.62; H, 3.80; $\mathrm{Cl}, 6.92 ; \mathrm{N}, 10.86 ; \mathrm{S}, 12.34$.

Ethyl 7-(1,3-Diphenyl-1H-pyrazol-4-yl)-2-oxo-3-phenyl3,5,6,7-tetra-hydro-2 $H$-thiopyrano[2,3- $d]$ thiazole-6-carboxylate $(7 \mathbf{e})$

Pale yellow crystals; yield $=61 \%$; mp $200{ }^{\circ} \mathrm{C} ;{ }^{1} \mathrm{H}-\mathrm{NMR}$ $\left(300 \mathrm{MHz}, \quad \mathrm{DMSO}-d_{6}\right) \quad \delta: 0.73\left(\mathrm{t}, 3 \mathrm{H}, J=6.9 \mathrm{~Hz}, \mathrm{CH}_{3}\right)$, 3.14-3.23 (m, 2H, H-5), 3.28 (t, 1H, J=5.1 Hz, H-6), 3.76 (q, $\left.2 \mathrm{H}, J=7.2 \mathrm{~Hz}, \mathrm{CH}_{2}\right), 4.70(\mathrm{~d}, 1 \mathrm{H}, J=4.2 \mathrm{~Hz}, \mathrm{H}-7), 7.33-7.96$ (m, 15H, Ar), 8.63 (s, 1H, pyrazole-H); IR $(\mathrm{KBr}) \mathrm{cm}^{-1}$ : 1720, 1660 (CO); MS m/z: $539\left(\mathrm{M}^{+}, 34.3 \%\right), 438$ (100\%), 346 (71.5\%), 319 (13.8\%), 256 (18.7\%), 173 (10.3\%), 104 (18.6\%), 77 (71.8\%). Anal. Calcd for $\mathrm{C}_{30} \mathrm{H}_{25} \mathrm{~N}_{3} \mathrm{O}_{3} \mathrm{~S}_{2}$ : C, 66.77; H, 4.67; N, 7.79; S, 11.88. Found: C, 66.60; H, 4.84; N, 7.56; S, 11.70.

Ethyl 7-(1-Phenyl-3-(4-methylphenyl)-1H-pyrazol-4-yl)-2oxo-3-phenyl-3,5,6,7-tetrahydro-2H-thiopyrano[2,3-d]thiazole-6-carboxylate (7f)

White crystals; yield $=77 \%$; mp $180^{\circ} \mathrm{C}$; ${ }^{1} \mathrm{H}-\mathrm{NMR}(300 \mathrm{MHz}$, DMSO- $\left.d_{6}\right) \delta: 0.80\left(\mathrm{t}, 3 \mathrm{H}, J=7.2 \mathrm{~Hz}, \mathrm{CH}_{3}\right), 2.39\left(\mathrm{~s}, 3 \mathrm{H}, \mathrm{CH}_{3}\right)$, 3.16-3.27 (m, 2H, H-5), 3.31 (t, $1 \mathrm{H}, J=5.4 \mathrm{~Hz}, \mathrm{H}-6), 3.78$ (q, $\left.2 \mathrm{H}, J=7.2 \mathrm{~Hz}, \mathrm{CH}_{2}\right), 4.80(\mathrm{~d}, 1 \mathrm{H}, J=4.2 \mathrm{~Hz}, \mathrm{H}-7), 7.41-8.01$ (m, 14H, Ar), 8.68 (s, 1H, pyrazole-H); IR (KBr) $\mathrm{cm}^{-1}: 1722$, 1663 (CO). Anal. Calcd for $\mathrm{C}_{31} \mathrm{H}_{27} \mathrm{~N}_{3} \mathrm{O}_{3} \mathrm{~S}_{2}$ (553.69): C, 67.25; H, 4.92; N, 7.59 S, 11.58. Found: C, 67.41; H, 4.74; N, 7.83; S, 11.78 .

Ethyl 7-(1-Phenyl-3-(4-methoxyphenyl)-1H-pyrazol-4-yl)-2oxo-3-phenyl-3,5,6,7-tetrahydro-2H-thiopyrano[2,3-d]thiazole-6-carboxylate $(\mathbf{7 g})$ 
White crystals; yield $=76 \%$; mp $215^{\circ} \mathrm{C} ;{ }^{1} \mathrm{H}-\mathrm{NMR}(300 \mathrm{MHz}$, DMSO- $\left.d_{6}\right) \delta$ : $0.78\left(\mathrm{t}, 3 \mathrm{H}, J=6.9 \mathrm{~Hz}, \mathrm{CH}_{3}\right), 3.15-3.22(\mathrm{~m}, 2 \mathrm{H}$, H-5), 3.28 (t, 1H, J=5.1 Hz, H-6), 3.78 (s, 3H, OCH $), 4.12$ (q, $\left.2 \mathrm{H}, J=6.9 \mathrm{~Hz}, \mathrm{CH}_{2}\right), 4.81(\mathrm{~d}, 1 \mathrm{H}, J=4.2 \mathrm{~Hz}, \mathrm{H}-7), 7.43-8.12$ (m, 14H, Ar), 8.72 (s, 1H, pyrazole-H); IR (KBr) cm ${ }^{-1}: 1721$, 1667 (CO). Anal. Calcd for $\mathrm{C}_{31} \mathrm{H}_{27} \mathrm{~N}_{3} \mathrm{O}_{4} \mathrm{~S}_{2}$ (569.69): C, 65.36; H, 4.78; N, 7.38; S, 11.26. Found: C, 65.18; H, 4.59; N, 7.61; S, 11.43 .

Ethyl 7-(1-Phenyl-3-(4-chlorophenyl)-1H-pyrazol-4-yl)-2oxo-3-phenyl-3,5,6,7-tetrahydro- $2 H$-thiopyrano[2,3-d]thiazole-6-carboxylate $(7 \mathbf{h})$

Pale yellow crystals; yield $=60 \%$; mp $223^{\circ} \mathrm{C}$; ${ }^{1} \mathrm{H}-\mathrm{NMR}$ $\left(300 \mathrm{MHz}, \quad \mathrm{DMSO}-d_{6}\right) \quad \delta: 0.81 \quad\left(\mathrm{t}, 3 \mathrm{H}, J=7.2 \mathrm{~Hz}, \mathrm{CH}_{3}\right)$, 3.18-3.29 (m, 2H, H-5), 3.38 (t, 1H, J=5.4 Hz, H-6), 4.09 (q, $\left.2 \mathrm{H}, J=7.2 \mathrm{~Hz}, \mathrm{CH}_{2}\right), 4.85(\mathrm{~d}, 1 \mathrm{H}, J=4.5 \mathrm{~Hz}, \mathrm{H}-7), 7.34-8.14$ (m, 14H, Ar), 8.76 (s, 1H, pyrazole-H); IR (KBr) cm ${ }^{-1}: 1720$, 1664 (CO). Anal. Calcd for $\mathrm{C}_{30} \mathrm{H}_{24} \mathrm{ClN}_{3} \mathrm{O}_{3} \mathrm{~S}_{2}$ (574.11): C, 62.76; H, 4.21; Cl, 6.17; N, 7.32; S, 11.17. Found: C, 62.60; H, 4.40; Cl, 6.0; N, 7.58; S, 11.36.

Preparation of 11-(1-Phenyl-3-aryl-1H-pyrazol-4-yl)-5hydroxy-3-phenyl-2H-benzo $[6,7]$ thiochromeno $[2,3-d]$ thiazole-2,10(3H)-dione $(9 a-d)$

General Procedure

A mixture of compounds $\mathbf{3 a}-\mathbf{d}(0.01 \mathrm{~mol})$ and 1,4-naphthoquinone $8(0.02 \mathrm{~mol})$ was refluxed for $1 \mathrm{~h}$ with catalytic amount of hydroquinone $(2-3 \mathrm{mg})$ to prevent polymerization process in $15 \mathrm{~mL}$ of glacial acetic acid. The reaction mixture was left overnight at room temperature. The solid precipitated was filtered off, washed with methanol and recrystallized from dimethylformamide.

11-(1,3-Diphenyl-1 $H$-pyrazol-4-yl)-5-hydroxy-3-phenyl- $2 H$ benzo[6,7]thio-chromeno[2,3-d] thiazole-2,10(3H)-dione (9a)

Pale yellow crystals; yield $=63 \% ; \mathrm{mp}>300^{\circ} \mathrm{C} ;{ }^{1} \mathrm{H}-\mathrm{NMR}$ $\left(300 \mathrm{MHz}, \mathrm{DMSO}-d_{6}\right) \delta: 7.07-7.94(\mathrm{~m}, 19 \mathrm{H}, \mathrm{Ar}), 8.70(\mathrm{~s}, 1 \mathrm{H}$, pyrazolyl-H), 10.0 (s, 1H, OH); IR (KBr) cm $\mathrm{cm}^{-1}: 3347(\mathrm{OH})$, 1668, 1664 (CO). Anal. Calcd for $\mathrm{C}_{35} \mathrm{H}_{21} \mathrm{~N}_{3} \mathrm{O}_{3} \mathrm{~S}_{2}$ (595.69): C, 70.57; H, 3.55; N, 7.05; S, 10.76. Found: C, 70.40; H, 3.73; N, $7.30 ; \mathrm{S}, 10.59$.

5-Hydroxy-3-phenyl-11-[1-phenyl-3-(4-phenylmethyl)- $1 H$ pyrazol-4-yl]-2H-benzo[6,7] thiochromeno[2,3-d]thiazole2,10(3H)-dione $(\mathbf{9 b})$

Whitish yellow crystals; yield $=59 \%$; mp $>300{ }^{\circ} \mathrm{C} ;{ }^{1} \mathrm{H}-\mathrm{NMR}$ $\left(300 \mathrm{MHz}, \mathrm{DMSO}-d_{6}\right) \delta: 2.20\left(\mathrm{~s}, 3 \mathrm{H}, \mathrm{CH}_{3}\right), 7.02-8.14(\mathrm{~m}, 18 \mathrm{H}$, Ar), 8.66 (s, 1H, pyrazolyl-H), 9.93 (s, 1H, OH); IR (KBr) $\mathrm{cm}^{-1}: 3345(\mathrm{OH}), 1670,1665$, (CO). Anal. Calcd $\mathrm{C}_{36} \mathrm{H}_{23} \mathrm{~N}_{3} \mathrm{O}_{3} \mathrm{~S}_{2}$ (609.72): C, 70.92; H, 3.80; N, 6.89; S, 10.52. Found: C, 70.74; H, 3.97; N, 6.65; S, 10.34 .

5-Hydroxy-11-[3-(4-methoxy phenyl)-1-phenyl-1 $H$ pyrazol-4-yl]-3-phenyl-2H-benzo[6,7]thiochromeno[2,3-d]thiazole-2,10(3H)-dione (9c)

Yellow crystals; yield $=68 \%$; mp $>300{ }^{\circ} \mathrm{C} ; \quad{ }^{1} \mathrm{H}-\mathrm{NMR}$ $\left(300 \mathrm{MHz}, \mathrm{DMSO}-d_{6}\right) \delta: 3.83\left(\mathrm{~s}, 3 \mathrm{H}, \mathrm{OCH}_{3}\right), 7.10-8.13(\mathrm{~m}$, $18 \mathrm{H}, \mathrm{Ar}), 8.68$ (s, 1H, pyrazolyl-H), 9.89 (s, 1H, OH); IR $(\mathrm{KBr}) \mathrm{cm}^{-1}$ : $3349(\mathrm{OH}), 1673,1666$, (CO). Anal. Calcd for $\mathrm{C}_{36} \mathrm{H}_{23} \mathrm{~N}_{3} \mathrm{O}_{4} \mathrm{~S}_{2}$ (625.72): C, 69.10; H, 3.71; N, 6.72; S, 10.25. Found: C, 69.29; H, 3.87; N, 6.95; S, 10.07.

11-(3-(4-Chlorophenyl)-1-phenyl-1H-pyrazol-4-yl)-5hydroxy-3-phenyl-2H-benzo[6,7] thiochromeno[2,3-d]thiazole-2,10(3H)-dione (9d)

Yellow crystals; yield $=70 \%$; $\mathrm{mp} \quad>300^{\circ} \mathrm{C} ; \quad{ }^{1} \mathrm{H}-\mathrm{NMR}$ $\left(300 \mathrm{MHz}, \mathrm{DMSO}-d_{6}\right) \delta: 7.07-8.18(\mathrm{~m}, 18 \mathrm{H}, \mathrm{Ar}), 8.71(\mathrm{~s}, 1 \mathrm{H}$,
pyrazolyl-H), 10.07 (s, 1H, OH); IR (KBr) cm $\mathrm{cm}^{-1}: 3343(\mathrm{OH})$, 1673, 1668 (CO). Anal. Calcd for $\mathrm{C}_{35} \mathrm{H}_{20} \mathrm{ClN}_{3} \mathrm{O}_{3} \mathrm{~S}_{2}$ (630.13): C, 66.71; H, 3.20; Cl; 5.63; N, 6.67; S, 10.18. Found: C, 66.89; H, 3.37 ; N, 6.44; S, 10.36 .

Crystallographic Data All measurements were made on a Nonius Kappa CCD area-detector diffractometer ${ }^{41)}$ using graphite-monochromated $\operatorname{Mo} K \alpha$ radiation $(\lambda=0.71073 \AA)$ and an Oxford Cryosystems Cryostream 700 cooler. The unit cell constants and an orientation matrix for data collection were obtained from a least-squares refinement of the setting angles of 53502 reflections in the range $4^{\circ}<2 \theta<55^{\circ}$. The mosaicity was $0.356(1)^{\circ}$. A total of 552 frames were collected using $\varnothing$ and $\omega$ scans with $K$ offsets, 30 s exposure time and a rotation angle of $1.0^{\circ}$ per frame, and a crystal-detector distance of $45.0 \mathrm{~mm}$. Data reduction was performed with HKL Denzo and Scalepack. ${ }^{42)}$ The intensities were corrected for Lorentz and polarization effects, and an absorption correction based on the multi-scan method $^{43)}$ was applied. The non-hydrogen atoms were refined anisotropically. All of the H-atoms were placed in geometrically calculated positions and refined by using a riding model where each $\mathrm{H}$-atom was assigned a fixed isotropic displacement parameter with a value equal to $1.2 \mathrm{U}_{\text {eq }}$ of its parent atom (1.5 $\mathrm{U}_{\mathrm{eq}}$ for the methyl group).

Crystal Data for $\mathbf{5 i}$

$\mathrm{C}_{36} \mathrm{H}_{26} \mathrm{~N}_{4} \mathrm{O}_{4} \mathrm{~S}_{2}, M=642.74$, size $0.13 \times 0.23 \times 0.25 \mathrm{~mm}^{3}$, orthorhombic, space group Pbca (\#61), $a=13.3045(2), b=15.7762(2)$, $c=29.74179(4) \AA, \alpha=90^{\circ}, \beta=90^{\circ}, \gamma=90^{\circ}, V=6174.7(1) \AA^{3}, Z=8$, $D_{\mathrm{c}}=1.383 \mathrm{~g} / \mathrm{cm}^{3}, F_{000}=2672, \mathrm{CCD}$ area detector, MoK $\alpha$ radiation, $\lambda=0.221 \AA, T=160(1) \mathrm{K}, 2 \theta_{\max }=4-55^{\circ}, 53502$ reflections collected, 74271 unique $\left(R_{\text {int }}=0.082\right)$. Final $R(F)[I>2 \sigma(I)$ reflections $]=0.0405, w R_{2}=0.1029, R$ indices based on 5201 reflections with $I>2 \sigma(I)$ (refinement on $F^{2}$ ), 417 parameters, $\mu=0.221 \mathrm{~mm}^{-1}$. CCDC 1006051 contains the supplementary crystallographic data for this paper. These data can be obtained free of charge at www.ccdc.cam.ac.uk/conts/retrieving. html [or from the Cambridge Crystallographic Data Centre (CCDC), 12 Union Road, Cambridge CB2 1EZ, UK; fax: +44 (0) 1223336 033; emaildeposit@ccdc.cam.ac.uk].

Acknowledgment The authors are very thankful to Prof. Dr. Khaled Abou-Hadeed in Institute of Organic Chemistry of University of Zürichfor running the X-ray crystallography of compound $\mathbf{5 i}$.

Conflict of Interest The authors declare no conflict of interest.

\section{References}

1) Singh S. P., Parmar S. S., Raman K., Stenberg V. I., Chem. Rev., 81, 175-203 (1981).

2) Veerasamy R., Rajak H., Suresh Kumar K., Kishore Agrawal R., Lett. Drug Des. Discov., 8, 82-86 (2011).

3) Saleh N. A. K., Saltani H. E. Al-Issa F. A. A., Melad A. S. G., J. Chin. Chem. Soc., 60, 1234-1240 (2013).

4) Dangar V., Dodia J. V., Shah V. R. Acta Chim. Pharm. Ind., 4, 90-96 (2014).

5) Day C., Diabet. Med., 16, 179-192 (1999).

6) Zheng W., Degterev A., Hsu E., Yuan J., Yuan C., Bioorg. Med. Chem. Lett., 18, 4932-4935 (2008).

7) Singh T., Sharma P. K., Kumar N., Dudhe R., J. Pharm. Negative Results, 4, 39-45 (2013)

8) Ragab F. A., Eid N. M., el-Tawab H. A., Pharmazie, 52, 926-929 
(1997).

9) Singh T., Sharma P. K., Mondalc S. C., Kumar N., J. Chem. Pharm. Res., 3, 609-615 (2011).

10) Kominsky D., Bednarczyk-Cwynar B., Vasylenko O., Kazakova O., Zimenkovsky B., Zaprutko L., Lesyk R., Med. Chem. Res., 21, 3568-3580 (2012).

11) Harkov S., Havrylyul D., Lesyk R., Chemistry \& Chemical Technology, 7, 381-389 (2013).

12) Geronikaki A. A., Lagunin A. A., Hadjipavlou-Litina D. I., Eleftheriou P. T., Filimonov D. A., Poroikov V. V., Alam I., Saxena A. K., J. Med. Chem., 51, 1601-1609 (2008).

13) De A., Jain S., Sharma P. C., Acta Pol. Pharm.-Drug Res., 67, 63-67 (2010).

14) Ahmed O., Salahuddin M., Rizwana I., Aleem M. A., Sharma P., Indo American J. of Pharm. Res., 3, 8121-8126 (2013).

15) Deep A., Jain S., Sharma P. C., Phogat P., Malhotra M., Med. Chem. Res., 21, 1652-1659 (2012).

16) Sugumaran M., Sethuvani S., Poornima M., J. Pharm. Biomed. Sci., 16, 1-4 (2012).

17) Sriram D., Yogeeshwari P., Ashok T. G., J. Pharm. Sci., 8, 426-429 (2005).

18) Rawal R. K., Prabhakar Y. S., Katti S. B., De Clercq E., Bioorg. Med. Chem., 13, 6771-6776 (2005).

19) Malipeddi H., Karigar A. A., Malipeddi V. R., Sikarwar M. S., Trop. J. Pharm. Res., 1, 611-620 (2012).

20) Havrylyuk D., Zimenkovsky B., Vasylenko O., Gzella A., Lesyk R., J. Med. Chem., 55, 8630-8641 (2012).

21) Kaminskyy D., Khyluk D., Vasylenko O., Zaprutko L., Lesyk R., Sci. Pharm., 79, 763-777 (2011).

22) Kaminskyy D., Zimenkovsky B., Lesyk R., J. Med. Chem., 44, 3627-3636 (2009).

23) Atamanyuk D., Zimenkovsky B., Lesyk R., J. Sulfur Chem., 29, 151-162 (2008)

24) Kaminskyy D., Vasylenko O., Atamanyuk D., Gzella A., Lesyk R., Synlett, 2011, 1385-1388 (2011).

25) Zelisko N., Atamanyuk D., Vasylenko O., Bryhas A., Matiychuk V.,
Gzella A., Lesyk R., Tetrahedron, 70, 720-729 (2014).

26) Kryshchyshyn A., Atamanyuk D., Lesyk R., Sci. Pharm., 80, 509529 (2012).

27) Chauhan A., Sharma P. K., Kaushik N., Inter. J. Chem. Tech. Res., 3, 11-17 (2011).

28) Priyadarsini P., Ujwala B., Rao C. V., Rao V. M., Der Pharmacia Letter, 4, 1123-1128 (2012).

29) Zheng Y., Zheng M., Ling X., Liu Y., Xue Y., An L., Gu N., Jin M., Bioorg. Med. Chem. Lett., 23, 3523-3530 (2013).

30) Ead H. A., Abdallah S. O., Kassab N. A., Metwally N. H., Saleh Y. E., Arch. Pharm., 320, 1227-1232 (1987).

31) Ead H. A., Abdallah S. O., Kassab N. A., Metwally N. H., Sulfur Letters, 9, 23-32 (1989).

32) Metwally N. H., Heterocycles, 75, 319-332 (2008).

33) Metwally N. H., J. Sulfur Chem., 28, 275-284 (2007).

34) Bianchi G., Gandolfi R., DeMichelli C. D., J. Chem. Res. (S), 6, ibid. (M), 1035 (1981).

35) Guarda V. L. M., Bosso C., Galdino S. L., Albuquerque J. F. C., Lima M. C. A., Silva J. B. P., Leite L. F. C., Pitta I. R., Spectroscopy, 18, 613-619 (2004).

36) Metwally N. H., J. Sulfur Chem., 35, 528-537 (2014).

37) Asche C., Mini Rev. Med. Chem., 5, 449-467 (2005).

38) Garuti L., Robert M., Pizzirani D., J. Med. Chem., 7, 481-489 (2007).

39) Lesyk R., Zimenkovsky B., Atamanyuk D., Jensen F., Kieć-Kononowicz K., Gzella A., Bioorg. Med. Chem., 14, 52305240 (2006).

40) Kira M. A., Abdel-Rahaman M. O., Gadalla K. Z., Tetrahedron Lett., 10, 109-110 (1969).

41) Kappa R., CCD Collect Software, Nonius BV, Delft, the Netherland (1999).

42) Otwinowski Z., Minor W., "Methods in Enzymology," Vol. 276, Part A, ed. by Carter C. W. Jr., Sweet R. M., Academic Press, New York, 1997, pp. 307-326.

43) Blessing R. H., Acta Crystallogr. A, 51, 33-38 (1995). 